\title{
Complejidad y Evolución en Sistemas de Conocimiento: Dispositivos de producción de complejidad en el sistema científico- técnico en Chile
} (2)

\author{
Complexity and evolution in knowledge Systems: complex \\ mechanism in chilean science and technology Systems
}

\section{Ronald Cancino Salas}

Universidad de la Frontera, Chile

\begin{abstract}
Resumen
El artículo, propone un análisis del problema de la complejidad y la evolución desde la óptica de la relación entre emergencia y jerarquía, especialmente en el campo de la modelación de sistemas de conocimiento. Se propone que desde una "epistemología evolucionaria", de corte complejo, es posible observar que el campo de la ciencia resulta particularmente interesante puesto que su modelación supone la observación (modelamiento) de las observaciones (estudios/intervenciones sobre la ciencia y la tecnología) que sobre las observaciones que produce la ciencia sobre la naturaleza/ sociedad. Es decir, permite analizar los acoplamientos/desacoplamientos entre dinámicas de variación/selección entre distintos niveles jerárquicos del sistema científico. Así, el problema de las transformaciones (morfogénesis) que implica el desarrollo científico-tecnológico en la actualidad, desde una óptica sistémica, compleja y evolucionaria, requerirían enfrentar o superar, algunos "obstáculos epistemológicos" instalados como dispositivos de observación de la relación ciencia sociedad. Se proponen al menos dos de estos dispositivos (neutralización del conocimiento y lógicas normativas asociadas a la semántica del desarrollo) y se propone una hipótesis que permitiría abordar el problema de la evolución de sistemas complejos, como la ciencia y la tecnología. Para modelar así la dinámica de transformaciones, si se permite, la dinámica evolutiva de sistemas de conocimiento, se propone el concepto- método de "dispositivos de producción de complejidad". Se revisa conceptualmente esta noción (jerarquía, dispositivos de intercambio, niveles de integración, arquitectura de la complejidad) para luego proponer 2 hipótesis sobre la complejidad del sistema científico tecnológico chileno y que se constituyen como agendas de investigación futura.
\end{abstract}

\begin{abstract}
The article proposes a problem analysis of the complexity and the evolution from the emergency and hierarchy relationship point of view, especially in the knowledge systems' modelling field. It is proposed that starting from an "evolutionary epistemology", of a complex nature, it is possible to observe that the science field turns out particularly interesting since its modelling includes the observations' (studies/ interventions about science and technology) observation (modelling) rather than observations that the science produces about nature/ society. That means, it allows analysing the connections/disconnections between variation/selection dynamics between different hierarchical levels of the scientific system. This way, the problem of the transformations (morphogenesis) that involves the scientifictechnological development currently, from a systemic, complex and evolutionary, point of view would require facing or overcoming some "epistemological obstacles" installed as observation mechanisms of the science-society relationship. Two of
\end{abstract}


these mechanisms are proposed at least (neutralization of the knowledge and rules logics associated to the development semantics) and a hypothesis is proposed that would allow dealing with the problem of complex systems evolution, like the science and the technology. To model the transformations dynamics, if it is allowed, the knowledge system evolutionary dynamics, the "complex production mechanisms" concept-method is proposed. This notion is conceptually revised (hierarchy, exchange mechanisms, integration levels, complexity architecture) to propose 2 hypotheses about the Chilean scientific-technological system complexity that are represented as future research agendas.

Palabras Clave: complejidad; epistemología evolucionaria; sistemas de conocimiento; políticas de ciencia y tecnología; sistema nacional de innovación.

Keywords: complexity; evolutionary epistemology; knowledge Systems; science and technology policies; national innovation Systems

\section{Introducción}

La ciencia, tecnología e innovación constituyen en la semántica de la transformación social hacia la "sociedad del conocimiento", el centro del problema tanto en términos explicativos como normativos. Se plantea que contando con buenos "diseños" de política (como la estrategia nacional de innovación, estrategias regionales de ciencia, tecnología e innovación, políticas sectoriales de fomento productivo y científico-tecnológico) sería posible transitar a aquella sociedad donde el conocimiento científico técnico podría agregar valor a la producción, como mecanismo para superar los déficit del patrón de baja especialización tecnológica dominante, y con ello, "tirar" a la sociedad hacia un "estadio" marcado por la centralidad del conocimiento. Con ello, se podría lograr una "complejidad" del sistema científico- técnico acorde a los requerimientos económicos y políticos actuales y de futuro. De hecho, si se observan los dilemas sociales que marcan la agenda pública, muchos de ellos tienen que ver con el modo como se propone resolver el avance científico- técnico.

El presente artículo, propone situar el problema en una óptica sistémica inspirada en Luhmann (1996), compleja (en la lógica de sistemas adaptativos complejos) y evolucionaria (en la óptica de la economía evolucionaria), que permita explicar los problemas de las prescripciones normativas (basadas en la construcción de mundos ideales) y neutralizadoras (desancladas de sus componentes ideológicos y socioculturales) que imperan en la actualidad. Se plantea lo siguiente: el análisis del sistema científico tecnológico actual - el Sistema Nacional de Innovación- según las aproximaciones desde la teoría de sistemas de innovación (Benavente, 2009) es un campo fértil para comprender el modo como se vincula el problema de la "emergencia", con el problema de la evolución de sistemas y, especialmente, el problema de la jerarquía como motor de transformación. Se trata de lo siguiente: si estamos en presencia de transformaciones sociales de fondo, como comprender la "naturaleza" de estos cambios?; nos referimos a cambios en configuraciones locales, o serían "estados emergentes"?; se trata de una verdadera "evolución" del sistema científico tecnológico? Se trata de una nueva semántica del progreso, o es posible identificar verdaderos dispositivos de transformación? De existir estos últimos, cuáles son?, que mecanismos explicativos podemos elaborar? Se propone para abordar ello lo siguiente: a partir del planteamiento central de Luhmann sobre la ciencia (1996) la descripción de un sistema científico supone (y esta sería el núcleo de la sociedad del conocimiento) no la descripción del estado del sistema (observación de primer orden), sino la observación de las observaciones que la ciencia hace sobre el mundo, y las comunicaciones que de ahí se despliegan, entonces la descripción de las transformaciones del sistema refiere a la descripción de las descripciones que del sistema científico se hacen, incluyendo aquellas que describen/prescriben el modo como se 
transformaría (o debería transformar) la sociedad. Se propone que, al incorporar tesis evolucionarias y abordando la noción de jerarquía, es posible identificar dispositivos de producción de complejidad social que estarían en la actualidad (y quién sabe, en el futuro) produciendo los principales espacios de transformación social que proponemos denominar sociocientíficas y sociotécnicas.

El artículo procede de la siguiente forma. La primera sección, aborda algunos problemas que impiden abordar en toda su extensión la complejidad que genera la ciencia, tecnología e innovación en la sociedad actual. Se aborda el problema de las descripciones normativas v/s la necesidad de explicaciones por mecanismos, así como la neutralización del conocimiento y la tecnología v/s la necesidad de comprensión de sistemas sociocientíficos o sociotécnicos. Se concluye con la siguiente hipótesis: la observación de la complejidad es la observación que emerge en las brechas entre los dispositivos de observación de lo "científico-técnico" y las transformaciones en marcha. La segunda sección, propone la noción de "dispositivos de producción de complejidad" como herramienta para observar las observaciones/acciones que de/sobre la ciencia y tecnología se hacen. Ello permitiría "modelizar" los dispositivos que en la actualidad producen complejidad sociocientífica y sociotécnica. Se argumenta teóricamente la noción de dispositivo como un mecanismo que se instala entre la noción de emergencia de segundo orden y jerarquía en la evolución de los sistemas. La tercera sección, propone algunos de los dispositivos que en la actualidad permiten comprender la estructura de la dinámica evolutiva de sistemas sociocientíficos y sociotécnicos en Chile. Se concluye con algunos planteamientos de una agenda de investigación en torno a la noción de máquinas de modelación de conocimiento.

\section{Obstáculos epistémicos para abordar la complejización sociocientífica y sociotécnica.}

La ciencia, tecnología e innovación han devenido en ser considerados un componente central a la hora de transitar hacia la sociedad del conocimiento. En este sentido, el debate actual chileno recorre varios senderos "controversiales": sobre la focalización tecnológicasectorial (lo que podemos denominar una "clusterización selectiva), sobre el diseño y la arquitectura institucional (controversias sobre la (des) centralización); sobre el nivel de los sistemas regionales de innovación (los anclajes e impactos territoriales), sobre los patrones de especialización tecnológica (las brechas y el impacto tecnológico), sobre las capacidades de acoplamiento del sistema universitario (controversias sobre su estatus público/privado y sus impactos), y, sobre la capacidad de acumulación e impactos del conocimiento científico (controversias sobre la eficiencia de individuos y redes). Estos debates, en la actualidad se estructuran en debates diríamos "parciales", o "segmentados: abordan las reglas locales de transformación del sistema científico, o de las relaciones de este con la sociedad. En su trasfondo, se han construido principalmente en una óptica económica (el impacto desigual en la dotación de factores de la producción y la multiplicación total de los factores) y normativa (en torno a visiones de futuro que inciden en la definición cambios y ajustes institucionales en el marco del debate sobre fallas de estado y fallas de mercado).

Ahora bien, ocurre que estas transformaciones en marcha, permiten indicar que estamos en presencia de una verdadera "transición de fase" no solo en el modo como se juegan los destinos del sistema científico-tecnológico, y con ello, parte de las transformaciones sociales, sino también, y fundamentalmente, obligan a pensar en el estatus de las observaciones que se realizan respecto de estas transformaciones. Se enfrenta aquí el primer problema evolutivo: si es posible aproximarse a un origen que haga comprensible el estado de las observaciones e irritaciones que se hacen a la ciencia y técnica, nos encontramos con lo siguiente: el sistema de Ciencia, Tecnología e Innovación chileno nace con un dilema en su seno. Se trata de una política cultural (anidada a la institucionalidad 
educativa chilena), o se trata de una política económica y tecnológica?. A este problema, le proponemos denominar "el doble vínculo" de la política de CTI en Chile (Cancino, 2009).

Nos encontramos entonces con el siguiente problema que instala la necesidad de pensar sistémicamente la evolución sociocientífica y sociotécnica: el sistema se constituye en operaciones heterorreferenciales que quieren hacer emerger operaciones autoreferenciales en el seno del sistema científico (verdad/no verdad) y tecnológico (útil/no útil). Es posible resolver esta paradoja?. El sistema científico entonces, se instala, y viene desarrollándose, intentando resolver el problema original: su constitución como sistema multijerárquico que no opera con las distinciones que se espera operen la dinámica autoorganizadora de cada sistema parcial. Si se observan los dilemas o "controversias" identificados, constituyen "fenómenos emergentes" que retroaccionan sobre la organización del sistema: la focalización tecnológica sectorial opera en la distinción natural/artificial en la emergencia de la lógica de los cluster (es natural la clusterización o se promueve su implementación?); la controversia sobre diseños institucionales opera en la distinción poder central/poder local, resolviéndose permanentemente en la primera parte de la distinción; la controversia regional, opera en la distinción territorial "basal" (en el sentido de comunicación basal de Wilke (2006) endógeno/exógeno en el operar de las prioridades; la controversia sobre la especialización tecnológica opera en la distinción profundización de brechas/superación de brechas; la controversia universitaria opera en la distinción público/privado en la transmisión del saber y en el acoplamiento/desacoplamiento del mundo del trabajo y la demanda empresarial (Chávez, 2010). Finalmente, la controversia sobre las capacidades de acumulación de conocimiento, opera en la distinción eficaz/ineficaz de la inversión.

De este modo, el problema central que se enfrenta es que este conjunto de distinciones coevolucionan instalando una urgente necesidad de comprensión de estas en su conjunto, pues es allí donde se juega la comprensión y "conducción" del sistema en su conjunto. Pues bien, este es el principal problema. No se cuenta en la actualidad con dispositivos de observación que permitan realizar este procedimiento como un todo. Dicho de otro modo, desde la ciencia es posible contar con un dispositivo de observación compleja de sus transformaciones?. Una pista es la siguiente: comprendemos a la ciencia y la tecnología como un sistema parcial de primer orden, de modo que se instala como dispositivo de observación la lógica normativa lineal del desarrollo y, en segundo término, se instala la lógica neutralizadora del conocimiento y la técnica:

Primero. El problema de las explicaciones normativas. En el seno de la semántica del desarrollo opera un dispositivo que observa la sociedad desde un horizonte deseado, desdoblando la observación de lo "real" en su relación con un cierto estado ideal de la sociedad que, evidentemente no existe. Franz Hinkelammert (1970) es quizás quien más claramente, hace casi 50 años ya, identifico este problema. Nos parece que desde una óptica sistémica y compleja podemos hoy mejor comprender los efectos del principio de "no factibilidad del orden trascendental". Ocurre que existen mecanismos en los cuales se desdobla la no factibilidad: las nociones de sujeto, de conocimiento, de intercambio, devienen en dispositivos que se observan como incompletos, no por su carácter sistémico, o por necesidad de observación de su emergencia, sino por comparación en relación al estado ideal supuesto. Veamos:

- El sujeto científico, atrapado entre dos lógicas. Los sujetos, por ejemplo, los científicos, son observados en su incompletitud en la semántica de la "torre de marfil"1: se acusa a los científicos indicándolos como sujetos que buscan solamente el despliegue de su interés científico v/s los intereses de la sociedad. Analizado sistémicamente, de que se trata?. Si la ciencia opera en sus propias

\footnotetext{
${ }^{1}$ Ver texto de HEBE VESSURI.
} 
distinciones, se necesita entonces que opere con distinciones exteriores?. La exterioridad implicaría que el saber se oriente por la utilidad...entonces se desarmaría el sistema científico, y el científico se transformaría en político. Y ello, cuando ocurre, reinstala la discusión desde su reverso: el científico no realiza su tarea de búsqueda de la verdad, sino que se moviliza "haciendo negocios". De que se trata entonces?. Luego, el científico si busca la verdad, lo hace en su derrotero científico, que debe conducir, y las líneas de investigación, en la semántica científica, pertenecen al propio investigador. Entonces, como se acopla en redes?. Publicar o hacer consultorías?. El sistema científico introduce regulaciones que dificultan el acoplamiento entre ambos: la universidad premia las publicaciones, el mercado las patentes o consultorías.

Los conocimientos y sus brechas en la óptica normativa: el conocimiento científico está siempre sometido a la incompletitud que se observa desde la lógica normativa. Ello opera en la heterorreferencia de la distinción saber/no saber, útil/no útil del conocimiento. En la actualidad, se distingue entre ciencia de base, de ciencia de base con orientación aplicada, como algo distinto del conocimiento aplicado, o tecnología. El sistema chileno, tiende a intentar desdoblar esta distinción en un ordenamiento institucional. De un lado, fondos orientados a la ciencia de base, de otro, fondos orientados a la aplicación. La noción de ciencia de base con orientación aplicada, emerge como solución intermedia para hacer tender el instrumental de la ciencia de base a su aplicabilidad. Es diríamos, una solución espuria: quiere instalar en el seno de la distinción verdad/no verdad, la distinción útil/ no útil. La heterorreferencia normativa del conocimiento, a este respecto opera del siguiente modo: se trata de identificar el estado del conocimiento científico al cual se debe llegar para lograr un cierto estado sistémico capaz de producir las innovaciones requeridas (brechas científico- técnicas en la semántica de la política científica y tecnológica). El salto cualitativo así se observa desde el punto de llegada. La pregunta es, cómo evoluciona el conocimiento desde puntos de llegada (su ideal normativo) si se cuenta con un sistema organizado en la alimentación de trayectorias que instalan ciclos recursivos de acumulación individual?. La lógica normativa aquí, impide observar el modo como observan los científicos: se procede por "path- dependence" (Dosi, 1982; Edquist, 1997), acumulación de trayectorias, se restringen progresivamente los "grados de libertad" a partir de lo que ya se viene desarrollando. Los científicos producen conocimiento a partir de lo que ya vienen produciendo, y no por una metaobservación desde un estado futuro ideal. El conocimiento así, no puede observarse desde un ideal, pues traicionaría su principio de base: la búsqueda de la verdad como búsqueda y no como una finalización total de la búsqueda.

El intercambio y la doble regulación: los mecanismos de regulación del sistema científico tecnológico, vienen organizados en lo que hemos denominado el "doble vínculo". La organización tiene dos estructuras superpuestas (no es posible diríamos sistémicamente). Con ello, las regulaciones regulan con dos orientaciones, finalistas, o normativas ambas: mejorar el estado "cultural" de la sociedad y solucionar los problemas "de agregación de valor" de los sistemas productivos. No es casual que la semántica de los problemas de la educación chilena se conciban como dificultades para el despliegue del conocimiento científico, pero a su vez, se identifiquen problemas de adecuación del conocimiento científico. Pareciera ser un problema en cascada: la educación produce sujetos poco aptos para la ciencia y técnica, la ciencia y técnica no produce sujetos aptos para el trabajo, el trabajo no produce competencias para mejorar la competitividad, la ciencia no produce mejoras de la competitividad, la falta de competitividad contiene el desarrollo científico-técnico....un mecanismo recursivo de producciones de contenciones de desarrollo en la óptica normativa. 
Segundo. La neutralización del conocimiento y las técnicas. En la literatura sociológica, antropológica y económica del conocimiento y la técnica, progresivamente se tiende hacia lo que se conoce como la "apertura de la caja negra" (Woolgar, 1991; Latour y Woolgar, 1985). Se observa aquí una brecha de conocimiento entre la concepción explicativa y normativa del proceder científico- técnico, que se intenta resolver con dos mecanismos, de primer orden uno (neutralizar) y relativista el segundo (constructivista). Veamos. Quizás en las controversias tecnológicas es donde mejor se observa ello. En los procesos de discusión sobre la factibilidad técnica de megaproyectos tecnológicos (centrales energéticas), todos los actores requieren poner en evidencia la relevancia del conocimiento científico que avala o rechaza tal o cual proyecto tecnológico. De hecho, el sistema de evaluaciones de impacto se sustenta sobre esta base: se analiza, desarmando los proyectos, en el conjunto de elementos que deben ser analizados en sus impactos ("a" -el elemento del proyecto- impacta a "b", una parte de la sociedad). Sobre esta "comunicación basal", se producen las comunicaciones siguientes: cada actor intenta dar cuenta de la verdad y eficacia del conocimiento que defiende y de la neutralidad de las técnicas que dan lugar a los análisis. Así, la evolución de las controversias hace coexistir semánticas: la neutralidad del conocimiento se asocia al funcionamiento de las instituciones, y la demanda por su desneutralización refiere a planteamientos constructivistas: habrían agentes que utilizan el conocimiento en su favor para intentar incidir en la resolución de las controversias (Pinch, 1997). Lo interesante comienza a desplegarse cuando se observa que en múltiples proyectos viene desarrollándose el mismo dispositivo: se generaliza la neutralización y la agenda constructivista. Paradojalmente, distintos agentes levantan distintas hipótesis sobre un mismo fenómeno. Entonces, será problema de alguno de estos agentes o problema del modo como se observa el problema de las controversias?. Lo extraño diríamos, es que se cuenta en la actualidad con instrumentales teóricos y metodológicos que permiten mejor observar y actuar en estos casos tipo. Se sabe que los agentes operan por "skills", pautas que orientan la acción (Nelson, Richard, 1987), y que las innovaciones emergen por acoplamientos/ desacoplamientos entre las estrategias de múltiples agentes que concurren en procesos de variación- selección de sus estrategias (op.cit.). La paradoja radica en que la dinámica de variación/selección está predicha de antemano, hay una selección "no evolutiva" que ante variaciones, gobierna el sistema. Se contiene así su evolución, volviéndose el sistema "subcomplejo", dicho de otro modo, se conoce de antemano el desenlace.

Conclusión 1. La complejidad social emerge por la incompletitud de la observación que introducen los dispositivos de observación disponibles en relación a las transformaciones que generan las acciones de esos dispositivos. Se trata entonces de lo siguiente: el instrumental de observación que disponemos, anclado a una mirada normativa que observa desde un estado social inexistente, requiere -para operar- simplificar la observación de las transformaciones que el propio sistema científico-técnico viene desplegando, ya sea como efectos de la implementación de políticas, como de transformaciones animadas por el propio mercado. Ello genera un escenario de dispositivos de observación "subcomplejos". Se requiere entonces, construir dispositivos de observación que den cuenta de la producción de complejidad social. Si se permite, el dilema es el siguiente: como pasar de la identificación de semánticas normativas/ neutralizantes a explicaciones plausibles de las transformaciones que operan en el despliegue científico- técnico de la sociedad chilena?

\section{Dispositivos de producción de complejidad social sociocientífica y sociotécnica}

Los planteamientos anteriores podrían ser sintetizados, metodológicamente, del siguiente modo: contamos con modelos propios de enfoques normativos de explicación de las dinámicas científico- técnicas cuya característica central es la exterioridad del modelo 
explicativo propuesto (observa desde un mundo deseado), para problemas que requieren una explicación teórica, (no causal- lineal) basada en la identificación de mecanismos, es decir, la observación de fenómenos emergentes. Con ello, se entiende que en la observación de lo social, se observan fenómenos que cuyas propiedades cobran autonomía en relación a los fenómenos o configuraciones locales que lo causan, pero retroaccionan sobre esos fenómenos (Dupuy,1998; Gilbert, 2002; Mascareño, 2009). Específicamente, en ciencia, Gilbert (1997) refiere a los "kene", unidades de conocimiento que "envuelven" a otras y que mutan en relación a los agentes que lo producen y las propiedades de campos científico técnicos (un set de capacidades tecnológicas en campos científico-técnicos, que emerge a partir de las habilidades y trayectorias del científico (Gilbert, 1997; 1999; 2002a). Por ejemplo, las líneas de investigación declaradas por los individuos científicos, no son las mismas que las líneas de investigación consideradas como un agrupamiento de producciones de conocimiento. Los individuos investigadores declaran una disciplina científica, pero las líneas de investigación tienden muchas veces a ser interdisciplinarias. Lo mismo ocurre con las redes de conocimiento. Los individuos conforman redes segmentadas (se vinculan con un número restringido de agentes), pero el accionar en redes genera una estructura de red en un campo científico que está más allá de las condicionantes locales que dan lugar a esas redes. Ahora bien, las líneas de investigación y las redes, concebidas como fenómeno emergente, inciden en el modo como los investigadores generan conocimientos, en el modo como generan redes y, con ello, en la conformación de una estructura científico- técnica en un campo determinado del conocimiento.

Entonces, para abordar las dinámicas de transformación científico-técnicas en la sociedad actual, se requiere observar dos clases de fenómenos: de un lado, el "set de conocimientos" que en/los campos en análisis emergen (como fenómenos emergentes) y, a su vez, el conjunto de fenómenos que hacen comprensible ese set de conocimientos. Esta relación es, desde nuestro punto de vista, fundamental. Nos encontramos aquí con un problema de niveles de observación, o "niveles de organización del conocimiento" (Atlan, 1991). Quizás, es la expresión de un viejo problema en las ciencias sociales, la relación entre sistemas de clasificación y sistemas de intercambio. Las clasificaciones emergen de interacciones, pero las clasificaciones cobran autonomía incidiendo en la estructura misma de los sistemas de intercambio² (Mauss, 1971).

En esta óptica, proponemos en esta sección que se requiere de la construcción de una modelación de los dispositivos que producen complejidad social. Una modelación consistiría en un la identificación de los mecanismos de producción de brechas de observación/acción que convocan a observar el modo como emergen fenómenos que producen complejidad sociocientífica y sociotécnica, y la comprensión del modo como los aspectos socioculturales son referenciados, producidos o heterorreferenciados en tanto dilemas que introducen complejidad a las dinámicas sociocientíficas y sociotécnicas que operan en la sociedad ${ }^{3}$.

\footnotetext{
${ }^{2}$ Durkheim y Mauss sugieren la noción de "emergencia de segundo orden", que es fundamental en la actualidad. Dice Mauss refiriéndose a las clasificaciones "Lo que caracteriza a estas últimas es que las ideas aparecen organizadas en ellas sobre un modelo que proporciona la sociedad. Pero una vez que existe esta organización de la mentalidad colectiva, es susceptible de volverse su causa y contribuir a modificarla" Emile Durkheim y Marcel Mauss (1903) De ciertas formas primitivas de clasificación. Contribución al estudio de las representaciones colectivas. En, Marcel Mauss, Institución y Culto, Obras II. Barral Editores, 1971. pág.31.

${ }^{3}$ Dice Atlan "La autoorganización implica necesariamente interacciones entre distintos niveles de integración, que al mismo tiempo son niveles diferentes de observación. En el marco del principio de "complejidad por ruido", describir la autoorganización como la utilidad de perturbaciones aleatorias para crear una complejidad funcional, equivalía a describir la creación de significaciones nuevas - y por lo tanto, aún desconocidas - en la información transmitida de un nivel a otro". Atlan, Henry. 1991. Pp. 67-68.
} 
La comprensión de la emergencia de complejidad sociocientífico y sociotécnica, requiere articular dos series de fenómenos: el estado y las transformaciones de las "estructuras de conocimiento" (con Gilbert, los kene) y el conjunto de fenómenos emergentes que podrían hacer comprensible esas estructuras de conocimiento.

Aquí, nos encontramos con un aspecto central: con Luhmann (1996), podemos identificar aquellos fenómenos emergentes propios de las cualidades autoorganizadoras del sistema científico y las comunicaciones que ellas generan. Así, por ejemplo, la lógica de doble dependencia de la política, las tensiones entre verdad/no verdad del conocimiento de base, la distinción útil/no útil respecto de la aplicación tecnológica, las distinciones saber/no saber - acceder/no acceder del sistema educativo, etc., pero se dificulta la comprensión de la estructura del conocimiento (podemos entender sus comunicaciones, pero no las dimensiones cognitivas del campo científico), que para nosotros, es un aspecto relevante. Por su parte, la sociología de la ciencia, especialmente la constructivista, propone modelos para comprender el modo como se abren/cierran controversias sociocientíficas (pero no los mecanismos que generan la controversia y los impactos sociotécnicos que generan), y la economía evolucionaria permite comprender el modo como en sistemas de innovación operan los "skills" en mecanismos de variación/selección, pero no el análisis global de las dinámicas de transformación societal.

De este modo, nos encontramos con la necesidad de construir una aproximación que integre desarrollos téorico-metodológicos provenientes de lo que podríamos denominar una "convergencia transdisciplinaria en ciernes", entre los abordajes de la complejidad "luhmanniana", la lógica de sistemas adaptativos complejos, algunos elementos de la sociología de la ciencia y la tecnología, y los debates de la economía evolucionaria.

La propuesta conceptual a este respecto, es la siguiente. La noción de jerarquía ${ }^{4}$ permite identificar los "niveles de integración" (Atlan, 1991) de la estructura de un sistema sociocientífico, que permitiría vincular los fenómenos emergentes modelados con las condiciones locales que dan lugar a esa emergencia. Esto es, dependiendo de la estructura jerárquica del sistema (diríamos, heterárquica, multijerárquica, o jerárquica en su sentido simple), las condiciones locales dan lugar a dispositivos de producción de complejidad que hacen emergen - valga la redundancia- fenómenos emergentes. Y sería entonces de este modo que es posible comprender la evolución de un sistema sociotécnico como la producción de complejidad sociocientífica y sociotécnica. De este modo, la noción de dispositivos de producción de complejidad viene a ocupar un rol metodológico relevante, en tanto permite identificar los mecanismos que auto-organizan la interacción entre niveles de integración (o dispositivos de intercambio -sujetos, objetos, mensajes-) y que hacen emerger la complejidad sociocientífica y sociotécnica y que, al producir emergencia, retroaccionan sobre los niveles inferiores del sistema sociocientífico y sociotécnico.

Se trata así de identificar, comprender y modelar, las relaciones entre los fenómenos emergentes que hacen comprensible la organización del sistema sociocientífico y sociotécnico y las estructuras de conocimiento que se han generado. La evolución así, se concibe como un proceso de producción de transformaciones locales que modifican la estructura global del sistema y que retroaccionan sobre los niveles inferiores, modificando las estructuras locales que producen transformaciones globales. Un dispositivo de producción de complejidad así, se concentra en la modelación de las transformaciones del sistema sociocientífico y sociotécnico identificando las "gradientes de complejidad" que emergen.

\footnotetext{
${ }^{4}$ Herbert Simon distingue entre jerarquía "formal" (aquel sistema organizado por dependencias funcionales de un centro), de "jerarquía": "Por sistema jerárquico entiendo aquel sistema formado por una serie de subsistemas relacionados entre sí cada uno de los cuales posee a su vez una estructura jerárquica hasta el nivel más inferior del subsistema elemental" (Simon, Herbert. 1996:219).
} 


\section{Conocimientos}

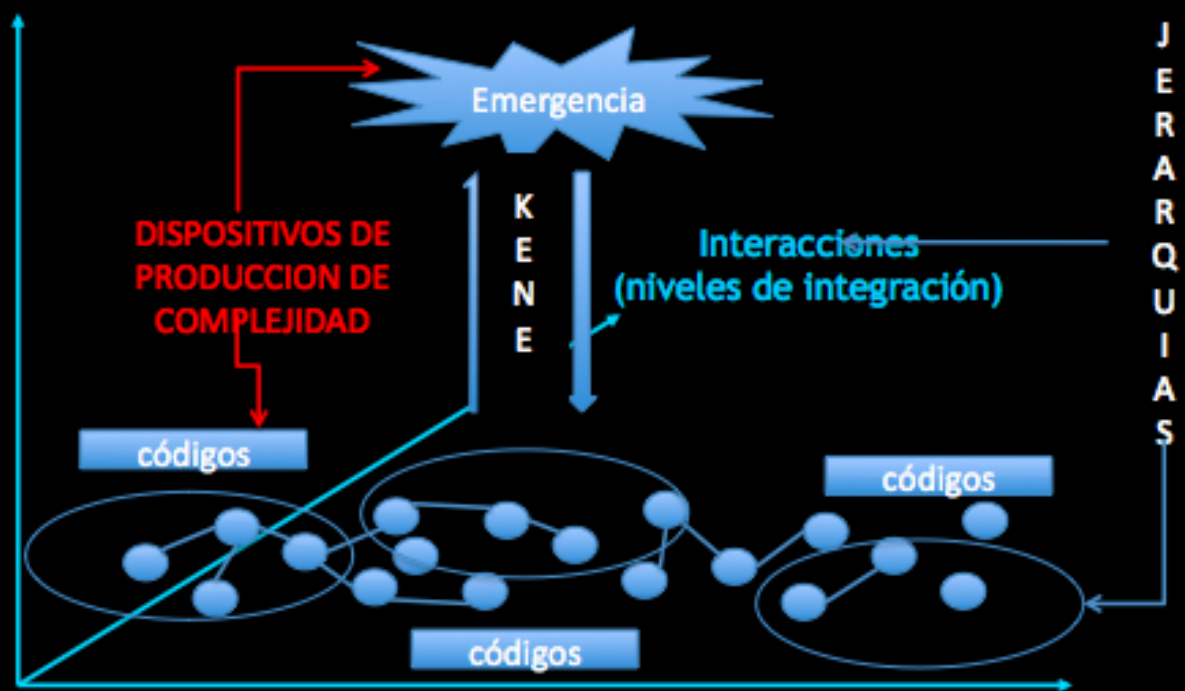

Tíempo

Dicho en síntesis. En una modelación de sistemas de ciencia, la integración está dada por las propiedades emergentes de su dinámica. Las propiedades emergentes emergen en mecanismos específicos, diríamos localizados, que vinculan un nivel macro/micro. Por eso, la noción de "dispositivos de producción de complejidad" puede operacionalizar tanto el rol de la jerarquía en la producción de fenómenos emergentes en sistemas complejos. Por ende, su evolución en el tiempo, y su operacionalización metodológica para la observación/modelación de la evolución de sistemas de conocimiento. Los dispositivos de producción de complejidad pueden ser comprendidos como Mecanismos que generan fenómenos emergentes que indican (son observaciones) espacios privilegiados de observación de interacciones entre dispositivos de intercambio (sujetos, objetos, mensajes). Dan cuenta de verdaderas "transiciones de fase" o puntos críticos de la evolución de un sistema complejo. Son fenómenos que indican gradientes evolutivas de complejidad. Finalmente, ofrecen un campo de distinciones en curso (procesos de producciones de distinciones en el campo en cuestión) que facilitan la identificación la "arquitectura del sistema".

\section{Conclusión 2: Hipótesis complejas para la modelación de dispositivos de producción de complejidad sociocientífica y sociotécnica en Chile}

Para concluir, se exponen algunas hipótesis organizadoras de una agenda de trabajo para la modelación.

Primero. La complejidad del Sistema de Ciencia y Tecnología en Chile emerge en las operaciones heterorreferenciales que se instalan en el proceso evolutivo de constitución del propio sistema (reconocible como evolución de la institucionalidad y de sus regulaciones) en las distinciones que introduce el problema del doble vínculo de la política (la política científica como problema de la cultura, o de la economía orientada al mejoramiento de la competitividad), así como en el proceso de acumulación de capacidades científicas (expresada en la tensión huella disciplinaria/requerimiento de los sectores de aplicación). Este proceso de coevoluciones puede ser comprendido como la 
HETEROGENEIDAD DINAMICA del Sistema de Ciencia y Tecnología, caracterizable como la articulación/no articulación de la producción científica (la semántica de la clasificación disciplinaria/ inter/transdisciplinaria) con los requerimientos sociales, productivos y de soluciones de innovación que a la ciencia se le demanda (semántica de la utilidad de la ciencia). Ello genera una producción interna de brechas que hacen coevolucionar capacidades y diseños institucionales que operan en los debates actuales en torno a la "unidad base de la ciencia" (individuo/grupo/red), brechas sectoriales (en torno a los sectores priorizados), territoriales (en torno a los sistemas regionales de innovación). Así mismo, en la actualidad la tendencia a la gestión de datos de investigación e información científica, tienden a modificar la naturaleza misma de la creación y distribución del conocimiento, desdoblándose las redes de investigación en redes de producción de conocimiento.

Segundo, la "medición" de dispositivos de producción de complejidad, puede ser desarrollado mediante el análisis de Trayectorias Científicas y Tecnológicas desde una óptica sistémica y compleja, entendidas como fenómenos emergentes medibles con técnicas cienciométricas de "segundo orden", como son la noción de proyecto de ciencia (membrana que articula instalando la distinción con el entorno del proyecto) y la noción de impacto (que produce la heterorreferencia respecto de que es lo clasificado/impactado (cultura/ producción) ${ }^{5}$. RM

\section{Bibliografía}

Atlan, H. (1991). Con razón y sin ella. Intercrítica de la ciencia y el mito. Barcelona: Tusquets.

Arthur, W. B. (1994). On the evolution of complexity. En Cowan, G. , Pines, d., Meltzer, D. (Edit.) Complexity. Metaphors, models and reality. Texas: Santa Fe Institute.

Benavente, J. M., Katz, J. \& Price, J.J. (2009). Economía evolucionista. Historia y desarrollos recientes. En Figueroa, Eugenio (edit). La teoría de la evolución y las ciencias económicas y sociales. Santiago de Chile: Editorial Universitaria.

Broncano, F. \& Peréz, A. R. (Coord.) (2009). La ciencia y sus sujetos. ¿Quienes hacen la ciencia en el siglo xxi?. México D.F.: Siglo XXI

Cancino, R. (2009). Sistemas Regionales de Innovación en Chile. Estado actual y escenarios de futuro. En von Baer (Ed.) Pensando Chile desde sus regiones. Temuco: Ediciones Universidad de la Frontera.

Chávez, M. (2011). Disyuntivas Universitarias; La Universidad entre la ciencia y la formación. Ponencia presentada a la Mesa Sociología de la Ciencia, la Tecnología y la Innovación. Congreso Chileno de Sociología, Valparaíso.

Díaz, D. (et.al) (2007). Exploraciones en Antropología y Complejidad. Buenos Aires: Grupo Antropocaos.

Dosi, G. (1982). Technological paradigms and technological trajectories. Research Policy, (11), 147- 162.

\footnotetext{
${ }^{5}$ El problema metodológico vinculado a los planteamientos teóricos expuestos, no son desarrollados en el presente trabajo.
} 
Edquist, C. (Ed.). (1997). Systems of Innovation: Technologies, Institutions and Organizations. London and Washington: Pinter Publishers.

Dupuy, J. P. \& Varela, F. (1998). Circularidades creativas: para la comprensión de los orígenes. En P. Watzlawick \& P. Krieg (Ed.), El Ojo del observador. Contribuciones al constructivismo. Barcelona: Gedisa.

Dupuy, J. P. (1998). El sacrificio y la Envidia. El liberalismo frente a la justicia social. Barcelona: Gedisa.

Gilbert, N. \& Troitzsch, K. (2006). Simulación para las ciencias sociales. Madrid: Mc Graw Hill.

Gilbert, N. (2002a). Varieties of emergence. Paper presented at the Agent 2002 Conference: Social agents: ecology, exchange, and evolution, Chicago.

Gilbert, N. (1997). A simulation of the structure of academic science. Sociological Research Online, 2(2)

Gilbert, N. (1999). First Draft of a Model of an Innovation Network, SEIN- Working Paper, June 1999.

Hinkelammert, F. (1970). Ideologías del desarrollo y dialéctica de la historia. Santiago de Chile: Universidad Católica de Chile.

Hodgson, G. (1995). Economía y Evolución. Madrid: Edit. Colegio de economistas de Madrid.

Kauffman, S. (2003). Investigaciones. Complejidad, autoorganización y nuevas leyes para una biología general. Barcelona: Tusquets.

Latour, B. \& Woolgar, S. (1995). La vida en el laboratorio. La construcción de los hechos científicos. Madrid: Alianza Universidad.

Leydesdorff, L. (2001). The Challenge of Scientometrics: The Development, Measurement, and Self-organization of Scientific Communications. USA: Universal Publishers/ uPUBLISH.com.

Luhmann, N. (1996). La Ciencia de la sociedad. México D. F.: Universidad Iberoamericana, Anthropos, ITESO.

Mascareño, A. (2010). Diferenciación y contingencia en América Latina. Santiago de Chile: Edit. Universidad Alberto Hurtado.

Mascareño, A. (2009). Medios Simbólicamente Generalizados y el Problema de la Emergencia. Cinta moebio, (36), 174- 197.

Nelson, R. (1987) Understanding technical change as an evolutionary process. Amsterdam: Edit. Columbia University.

Pinch, T. (1997). La construcción social de la tecnología: una revisión. En Santos, M. J. \& Díaz Cruz, R. (comp.), Innovación tecnológica y procesos culturales. Nuevas Perspectivas teóricas. México D. F.: Fondo de Cultura Económica. 
Prigogine, I. (1997). Tan solo una ilusión. Una exploración del orden al caos. Barcelona: Tusquets.

Simon, H. (1996). Las ciencias de lo artificial. Granada: Edit. Comares.

Vivanco, M. (2010). Sociedad y complejidad. Del discurso al modelo. Santiago de Chile: Edic. Universidad de Chile.

Wagensberg, J. \& Agustí, J. (Edit.) (1998). El progreso, ¿un concepto acabado o emergente?. Barcelona: Edit. Tusquets.

Willke, H. (2006). La transformación de la democracia como modelo de orientación de las sociedades complejas. Estudios Publicos, (102), 179- 201.

Woolgar, S. (1991). Abriendo la caja negra. Barcelona: Edit. Anthropos.

\section{Sobre el autor}

Ronald Cancino es Académico del departamento de Ciencias Sociales de la Universidad de La Frontera. Actualmente se desempeña como Investigador en el Centro de Investigaciones Sociológicas, perteneciente al Programa de Estudios de la Ciencia de la misma universidad y es alumno del Doctorado en Ciencias Sociales de la Universidad de Chile.

rcancino@ufro.cl

\section{Contacto}

Universidad de La Frontera

Dirección de Investigación

Avenida Francisco Salazar 01145, Temuco - Chile

Fono: (56) (45) 325096

Fax: (56) (45) 734023

Recibido: Julio/201 1

Aceptado: Agosto/201 1

Artículo disponible en: http:// www.facso.uchile.cl/publicaciones/mad/25/ cancino04.pdf 